ARTICLE

\title{
Directed synthesis of a hybrid improper magnetoelectric multiferroic material
}

Tong Zhu', Fabio Orlandi (10 2 , Pascal Manuel (1) 2, Alexandra S. Gibbs (D) 2, Weiguo Zhang ${ }^{3}$, P. Shiv. Halasyamani (i) ${ }^{3} \&$ Michael A. Hayward (ib ${ }^{1 \times}$

Preparing materials which simultaneously exhibit spontaneous magnetic and electrical polarisations is challenging as the electronic features which are typically used to stabilise each of these two polarisations in materials are contradictory. Here we show that by performing low-temperature cation-exchange reactions on a hybrid improper ferroelectric material, $\mathrm{Li}_{2} \mathrm{SrTa}_{2} \mathrm{O}_{7}$, which adopts a polar structure due to a cooperative tilting of its constituent $\mathrm{TaO}_{6}$ octahedra rather than an electronically driven atom displacement, a paramagnetic polar phase, $\mathrm{MnSrTa}_{2} \mathrm{O}_{7}$, can be prepared. On cooling below $43 \mathrm{~K}$ the $\mathrm{Mn}^{2+}$ centres in $\mathrm{MnSrTa}_{2} \mathrm{O}_{7}$ adopt a canted antiferromagnetic state, with a small spontaneous magnetic moment. On further cooling to $38 \mathrm{~K}$ there is a further transition in which the size of the ferromagnetic moment increases coincident with a decrease in magnitude of the polar distortion, consistent with a coupling between the two polarisations.

\footnotetext{
${ }^{1}$ Department of Chemistry, University of Oxford, Inorganic Chemistry Laboratory, Oxford, UK. ${ }^{2}$ ISIS Facility, Rutherford Appleton Laboratory, Chilton, UK.

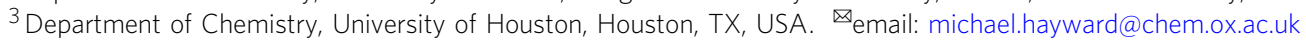


M agnetoelectric multiferroic materials ${ }^{1,2}$ - those which exhibit spontaneous and coupled magnetic and electrical polarisations-offer the prospect of preparing a range of devices for the manipulation and storage of digital information, including low-power nonvolatile random-access memories ${ }^{3,4}$. However, the preparation of magnetoelectric multiferroic materials is hampered by the contrasting electronic structures typically required for ferroelectric and ferromagnetic behaviour ${ }^{5}$. Specifically, the noncentrosymmetric, polar crystal structures, which are a prerequisite for ferroelectric materials $s^{6,7}$, are typically stabilised by second-order Jahn-Teller distortions ${ }^{8}$ of either $\mathrm{d}^{0}$ transition metals $\mathrm{s}^{9-11}$ or $n \mathrm{~s}^{2}$ post-transition metals ${ }^{12-15}$, and these closed-shell electronic configurations are not consistent with magnetism.

The "hybrid improper" (HIP) mechanism offers an alternative method for inducing the noncentrosymmetric polar crystal structures necessary for ferroelectric behaviour ${ }^{16-20}$. In this mechanism, two nonpolar structural distortions, typically the low-energy tilts of $B_{0}$ octahedral units in $A_{3} B_{2} \mathrm{O}_{7}$ and $A^{\prime}$ $A B_{2} \mathrm{O}_{7}$ layered perovskite phases, combine to break the inversion symmetry of the host framework and couple to a third polardistortion mode, leading to a spontaneous electrical polarisation $^{21-28}$. A key feature of the trilinear coupling between the three distortion modes is that the polar-distortion mode is not the primary order parameter of the ferroelectric-phase transition of this class of material and is unstable in the absence of the two nonpolar distortions, hence the improper label.

As the HIP mechanism does not rely on the presence of $\mathrm{d}^{0}$ or $n s^{2}$ diamagnetic ions, it should be easier, in principle, to combine magnetic behaviour with this class of ferroelectric. Indeed, one of the first materials predicted to exhibit HIP ferroelectric behaviour, $\mathrm{Ca}_{3} \mathrm{Mn}_{2} \mathrm{O}_{7}{ }^{16}$, adopts a canted antiferromagnetic state (weak ferromagnet) below $115 \mathrm{~K}^{29}$, in which magnetoelectric coupling has been observed ${ }^{24,30}$, in line with theory predictions ${ }^{16}$.

However, further investigation of the Ruddlesden-Popper and Dion-Jacobson phases that exhibit HIP ferroelectric behaviour reveals that large $B$-site cations are needed to stabilise the highly distorted perovskite frameworks required for the HIP mechanism to function ${ }^{21-27}$. When combined with the high $B$-site charges needed in these frameworks, this size requirement rules out the inclusion of the vast majority of paramagnetic transition-metal cations, and to date, the only candidate magnetoelectric multiferroic materials based on the HIP mechanism are $\mathrm{Ca}_{3} \mathrm{Mn}_{2} \mathrm{O}_{7}{ }^{16,24,30}$ and $\left[\mathrm{Ca}_{0.69} \mathrm{Sr}_{0.6} \mathrm{~Tb}_{1.85} \mathrm{Fe}_{2} \mathrm{O}_{7}\right]_{0.85}\left[\mathrm{Ca}_{3} \mathrm{Ti}_{2} \mathrm{O}_{7}\right]_{0.15}$, the latter of which uses the introduction of some large diamagnetic $\mathrm{Ti}^{4+}$ ions onto the perovskite $B$ site, via alloying the magnetic iron phase with diamagnetic $\mathrm{Ca}_{3} \mathrm{Ti}_{2} \mathrm{O}_{7}$, to help stabilise the required distorted perovskite framework.

Recently we have been using the facile cation-exchange chemistry of the $A^{\prime} A B_{2} \mathrm{O}_{7}$ Dion-Jacobson phases to tune the structural distortions of this family of materials to induce HIP ferroelectric behaviour ${ }^{20,27,32-35}$. Here we describe how this cation-exchange chemistry can be used to substitute paramagnetic cations onto the $A$-sites of HIP ferroelectric Ruddlesden-Popper oxides to yield an additional class of magnetoelectric multiferroic materials.

\section{Results and discussion}

$\mathrm{Li}_{2} \mathrm{SrTa}_{2} \mathrm{O}_{7}$ was prepared by a ceramic synthesis route from a combination of $\mathrm{Li}_{2} \mathrm{CO}_{3}, \mathrm{SrCO}_{3}$, and $\mathrm{Ta}_{2} \mathrm{O}_{5}$. Neutron powder diffraction (NPD) data indicate that $\mathrm{Li}_{2} \mathrm{SrTa}_{2} \mathrm{O}_{7}$ adopts a polar a $\mathrm{a}^{-} \mathrm{c}^{+} / \mathrm{a}^{-} \mathrm{a}^{-} \mathrm{c}^{+}$distorted, $n=2$ Ruddlesden-Popper structure, described in space group $A 2_{1} a m$, in which the lithium cations reside in pseudo tetrahedral coordination sites between the $\mathrm{SrTa}_{2} \mathrm{O}_{7}$ perovskite double sheets (Fig. 1a). Particle-sizedependent optical SHG data collected at room temperature ${ }^{36}$ (Fig. 1c) are consistent with this noncentrosymmetric structure, in contrast to previous reports ${ }^{37}$. Analysis of the structure of $\mathrm{Li}_{2} \mathrm{SrTa}_{2} \mathrm{O}_{7}$ reveals that it can be related to the undistorted aristotype Ruddlesden-Popper structure (space group $I 4 / \mathrm{mmm}$ ) by the application of the $\mathrm{X}_{3}{ }^{-}$and $\mathrm{X}_{2}{ }^{+}$distortion modes that tilt the $\mathrm{TaO}_{6}$ octahedra (Fig. 1a), and the $\Gamma_{5}{ }^{-}$polar-distortion mode, consistent with trilinearly coupled hybrid improper ferroelectric behaviour ${ }^{16}$.

$\mathrm{MnSrTa}{ }_{2} \mathrm{O}_{7}$ was prepared by reacting $\mathrm{Li}_{2} \mathrm{SrTa}_{2} \mathrm{O}_{7}$ with $\mathrm{MnCl}_{2}$ at $375^{\circ} \mathrm{C}$ under an inert atmosphere to prevent the conversion of $\mathrm{MnCl}_{2}$ to $\mathrm{MnO}$ or $\mathrm{Mn}_{3} \mathrm{O}_{4}$. NPD data (HRPD instrument) collected from the cation-exchanged material at room temperature were initially indexed using a modified unit cell based on that of $\mathrm{Li}_{2} \mathrm{SrTa}_{2} \mathrm{O}_{7}$ and fit using a structural model based on $\mathrm{Li}_{2} \mathrm{SrTa}_{2} \mathrm{O}_{7}$
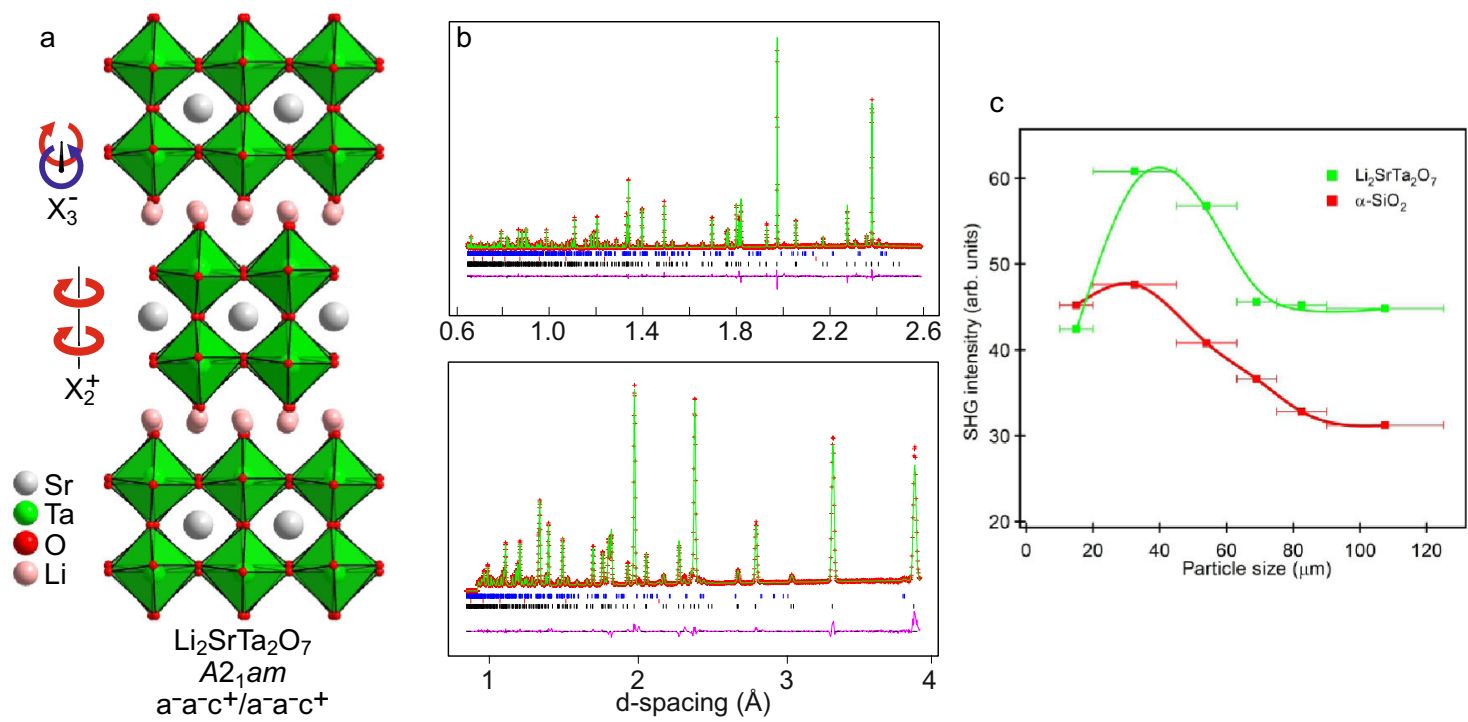

Fig. 1 The polar oxide $\mathrm{Li}_{2} \mathrm{SrTa}_{\mathbf{2}} \mathbf{O}_{\mathbf{7}}$. a The polar crystal structure of $\mathrm{Li}_{2} \mathrm{SrTa}_{2} \mathrm{O}_{7}$ viewed down the [110] direction. $\mathbf{b}$ Observed (red crosses), calculated (green line), and difference (purple line) plots from the structural refinement of $\mathrm{Li}_{2} \mathrm{SrTa}_{2} \mathrm{O}_{7}$ against NPD data. Black, red, and blue tick marks indicate peak positions for the majority phase, the vanadium sample holder and a small quantity of $\mathrm{Li}_{3} \mathrm{TaO}_{4}$, respectively. c Particle-size dependent $\mathrm{SHG}$ data from $\mathrm{Li}_{2} \mathrm{SrTa}_{2} \mathrm{O}_{7}$ compared with an $\alpha-\mathrm{SiO}_{2}$ standard. 

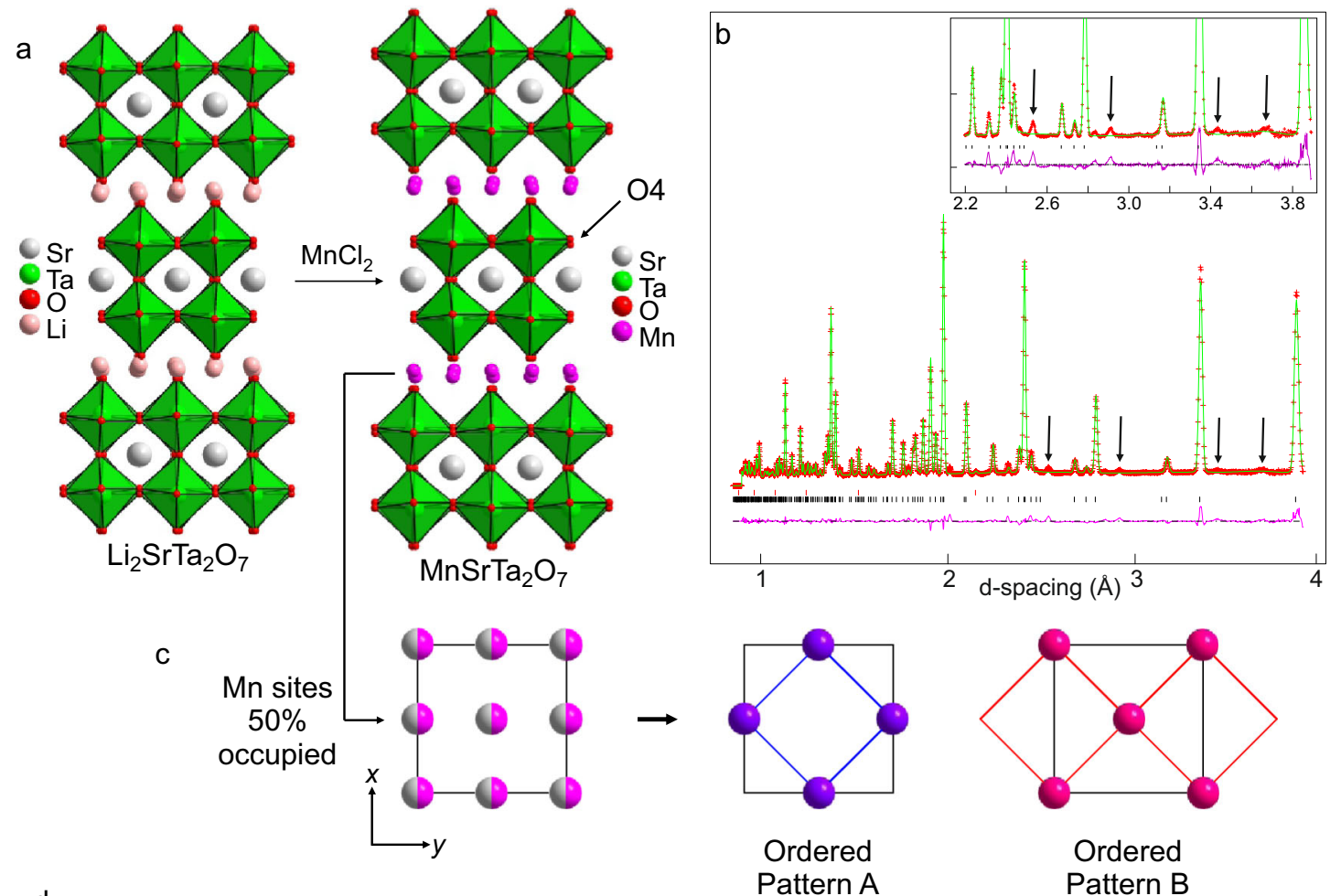

d

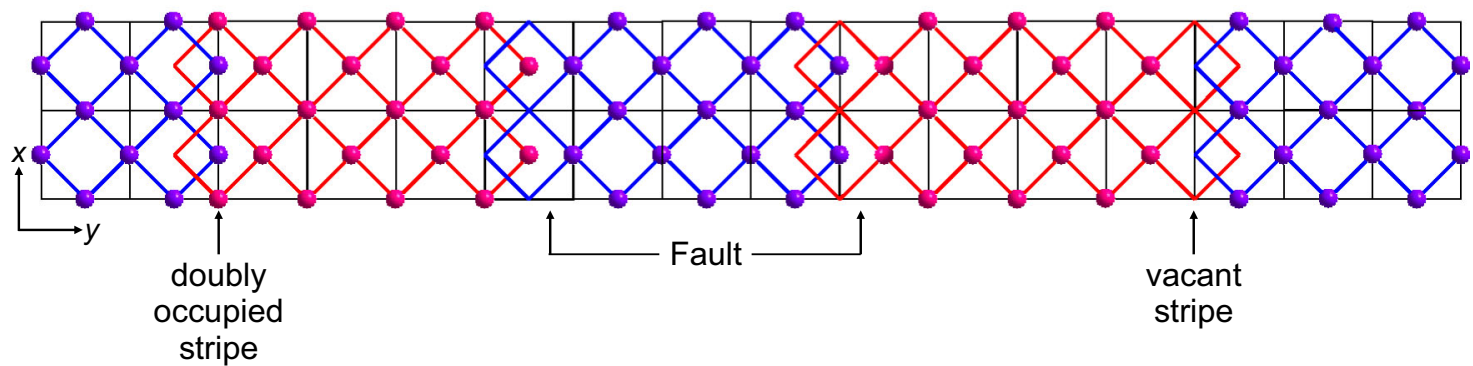

Fig. 2 Polar, incommensurate structure of $\mathbf{M n S r T a}_{\mathbf{2}} \mathbf{O}_{\mathbf{7}}$. a Reaction with $\mathrm{MnCl}_{2}$ converts $\mathrm{Li}_{2} \mathrm{SrTa}_{2} \mathrm{O}_{7}$ into $\mathrm{MnSrTa}_{2} \mathrm{O}_{7}$ via topochemical $\mathrm{Mn}$-for-Li cation exchange. b Observed (red crosses), calculated (green line), and difference (purple line) plots from refinement of an A2 ${ }_{1}$ am symmetry structural model against NPD data collected from $\mathrm{MnSrTa}_{2} \mathrm{O}_{7}$ at $300 \mathrm{~K}$. A series of peaks (marked by arrows) are not fit by this model and can only be indexed with an incommensurate unit cell. c Half occupancy of the pseudo tetrahedral coordination sites by Mn ${ }^{2+}$ allows two different local chequerboard orderings. d An approximate representation of the incommensurate intergrowth of the two Mn-ordering patterns, which propagates along the crystallographic $y$ axis. Locally ordered regions are joined by doubly occupied/vacant stripes or "faults" which run parallel to the $x$ axis.

(space group $A 2{ }_{1} \mathrm{am}$ ), but with the $\mathrm{Li}^{+}$cations replaced by a $50 \%$ occupancy of $\mathrm{Mn}^{2+}$ ions, as shown in Fig. 2a. Refinement of this model gave a good fit to the NPD data, with a refined Mn occupancy of $0.496(6)$, consistent with the stated chemical formula, and the observed SHG activity (0.26 times $a-\mathrm{SiO}_{2}$, Supplementary Fig. 2) of the material is consistent with the noncentrosymmetric structure, described in detail in the Supplementary Information.

Close inspection of the fit of this commensurate model to the NPD data revealed a series of weak diffraction peaks that could not be indexed by this model and that could not be assigned to impurity phases (Fig. 2b). However, these additional diffraction features could be indexed using an incommensurate unit cell in superspace group $A 2{ }_{1} a m(0 \beta 0) 000$ with a propagation vector $\mathbf{q}=(0,0.86,0)$ compared with the previously refined commensurate $A 2{ }_{1}$ am symmetry unit cell-a situation that is clearer in a further NPD data set collected at $200 \mathrm{~K}$ using the WISH diffractometer.

A series of test structural models were constructed and refined against the data to establish which components of the
$\mathrm{MnSrTa}_{2} \mathrm{O}_{7}$ framework led to the structural modulation. As shown in Fig. $2 \mathrm{c}$, the $\mathrm{Mn}^{2+}$ cations can adopt a chequerboardordered arrangement within the tetrahedral coordination sites, analogous to the arrangement of $\mathrm{Na}^{+}$cations in $\mathrm{NaNdNb}_{2} \mathrm{O}_{7}{ }^{27}$, resulting in an array of apex-linked $\mathrm{MnO}_{4}$ units, which maximises the Mn-Mn separation. However, there are two distinct ways of ordering the Mn cations (A and B), which are simply related by switching the occupied and unoccupied tetrahedral sites.

It was found that the best fit to the NPD data was achieved using a model with a Crenel modulation of the $\mathrm{Mn}^{2+}$ occupancy, which corresponds to an incommensurate intergrowth of these two ordering patterns, as shown approximately in Fig. 2d, and described in detail in the Supplementary Information ${ }^{38,39}$. In addition, there is a coupled modulation of the "equatorial" O4 oxide ions that are adjacent to the tetrahedral coordination sites (Fig. 2a), and which move in response to the presence or absence of an $\mathrm{Mn}^{2+}$ cation in the neighbouring coordination site. The resulting modulated structure is noncentrosymmetric and polar, consistent with the observed room-temperature SHG activity of 

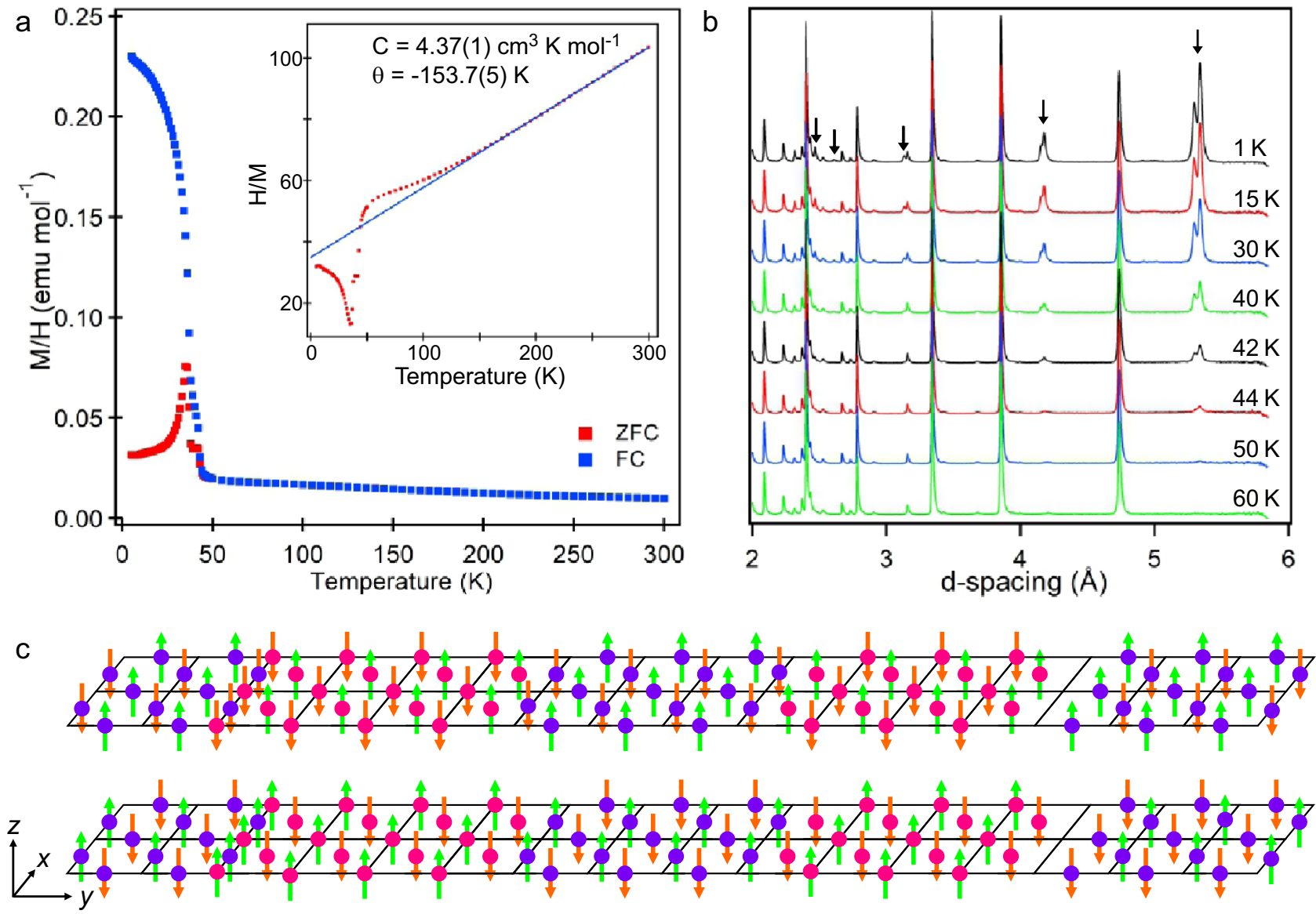

Fig. 3 Magnetic behaviour of $\mathbf{M n S r T a}_{2} \mathbf{O}_{7}$. a Zero-field-cooled and field-cooled magnetisation data collected from $\mathrm{MnSrTa}_{2} \mathrm{O}_{7}$, the inset shows the fit to the Curie-Weiss law in the range $150<T / \mathrm{K}<300$. b NPD data collected at indicated temperatures from $\mathrm{MnSrTa}_{2} \mathrm{O}_{7}$. Arrows mark magnetic scattering peaks. c The refined magnetic structure of $\mathrm{MnSrTa}_{2} \mathrm{O}_{7}$. Pink and purple spheres represent $\mathrm{Mn}$ cations using the colour scheme from Fig. 2 , green and orange arrows indicate the direction of the ordered magnetic moments.

the phase, with the electrical polarisation aligned parallel to the crystallographic $x$ axis.

The incommensurate modulated ordering of the $\mathrm{Mn}^{2+}$ cations over the two pseudotetrahedral coordination sites can be rationalised by noting that the commensurate, chequerboard ordering of the Mn cations, which maximises the Mn-Mn separation, is symmetry-incompatible with the polar $\mathrm{a}^{-} \mathrm{a}^{-} \mathrm{c}^{+} / \mathrm{a}^{-} \mathrm{a}^{-} \mathrm{c}^{+}$tilting of the $\mathrm{TaO}_{6}$ octahedra. This suggests that the observed modulated ordering pattern of the $\mathrm{Mn}$ cations is the optimum compromise between these two structural components ${ }^{32}$.

Zero-field-cooled (ZFC) and field-cooled (FC) magnetisation data collected from $\mathrm{MnSrTa}_{2} \mathrm{O}_{7}$ (Fig. 3a) can be fit by he Curie-Weiss law in the range $150<T / \mathrm{K}<300$ to yield a Curie constant of $\mathrm{C}=4.37(1) \mathrm{cm}^{3} \mathrm{~K} \mathrm{~mol}^{-1}$ consistent with the value expected for $S=5 / 2, \mathrm{Mn}^{2+}$ cations. On cooling below $43 \mathrm{~K}$, the ZFC and FC data diverge sharply, with field-dependent data exhibiting weak hysteresis at $5 \mathrm{~K}$.

NPD data collected at $1.5 \mathrm{~K}$ (Fig. 3b) show a series of strong magnetic Bragg peaks that can be indexed by a single magnetic propagation vector, $\mathbf{k}=(0,0,0)$, of the incommensurate crystallographic unit cell. The best fit to the magnetic diffraction data was achieved using a model described in magnetic space group $A 2_{1} a^{\prime} m^{\prime}(0 \beta 0) 000$, shown in Fig. $3 c$, in which the Mn moments $\left(4.06(1) \mu_{\mathrm{B}}\right.$ at $\left.1.5 \mathrm{~K}\right)$ are aligned parallel to the $z$-axis and ordered in an approximate G-type antiferromagnetic structure that is then perturbed by the modulated $\mathrm{Mn}$ cation order. During the refinement of the magnetic model, the crystallographic model was also simultaneously refined, and the structural modulation vector $\mathbf{q}=(0,0.864,0)$ was observed to be unchanged.

Magnetisation data indicate that there is a small ferromagnetic component (canting) to the magnetic structure $(0.04 \mu \mathrm{B}$ per $\mathrm{Mn}$ at $5 \mathrm{~K})$. The magnetic space group $\left(A 2_{1} a^{\prime} m^{\prime}(0 \beta 0) 000\right)$ allows a ferromagnetic component parallel to the $x$ axis, consistent with this observation. Furthermore, coupling between the antiferromagnetic order (which transforms as the $\mathrm{mX}_{3}{ }^{+}$irreducible representation) and the $\mathrm{X}_{2}{ }^{+}$structural distortion stabilises a weak ferromagnetic moment (which transforms as the $\mathrm{m \Gamma}_{5}{ }^{+}$irreducible representation) via a trilinear invariant, as described in the Supplementary information; thus, the magnetic structure of $\mathrm{MnSrTa}_{2} \mathrm{O}_{7}$ is required to have a weak ferromagnetic component by symmetry. However, the small size of this ferromagnetic component means that it could not be observed in the NPD data.

The temperature dependence of the ordered moment obtained from NPD data can be fitted by a power law, $M=M_{0}\left(1-\frac{T}{T_{N}}\right)^{\beta}$, to yield a Néel temperature of $T_{\mathrm{N}}=43.5(1) \mathrm{K}$, as shown in Fig. 4b. However, it can be seen from the data in Fig. $3 \mathrm{~b}$ that magnetic scattering persists above this temperature. Close inspection of the data reveals that above $43 \mathrm{~K}$, the magnetic scattering peaks broaden significantly as they diminish in intensity, and diffuse scattering features appear in the data, as highlighted in the Supplementary Information. This peak broadening/ diffuse scattering is attributed to the persistence of strong $2 \mathrm{D}$ magnetic interactions in the $x y$ plane above $T_{\mathrm{N}}$. Indeed, the value 


$$
\text { a }
$$
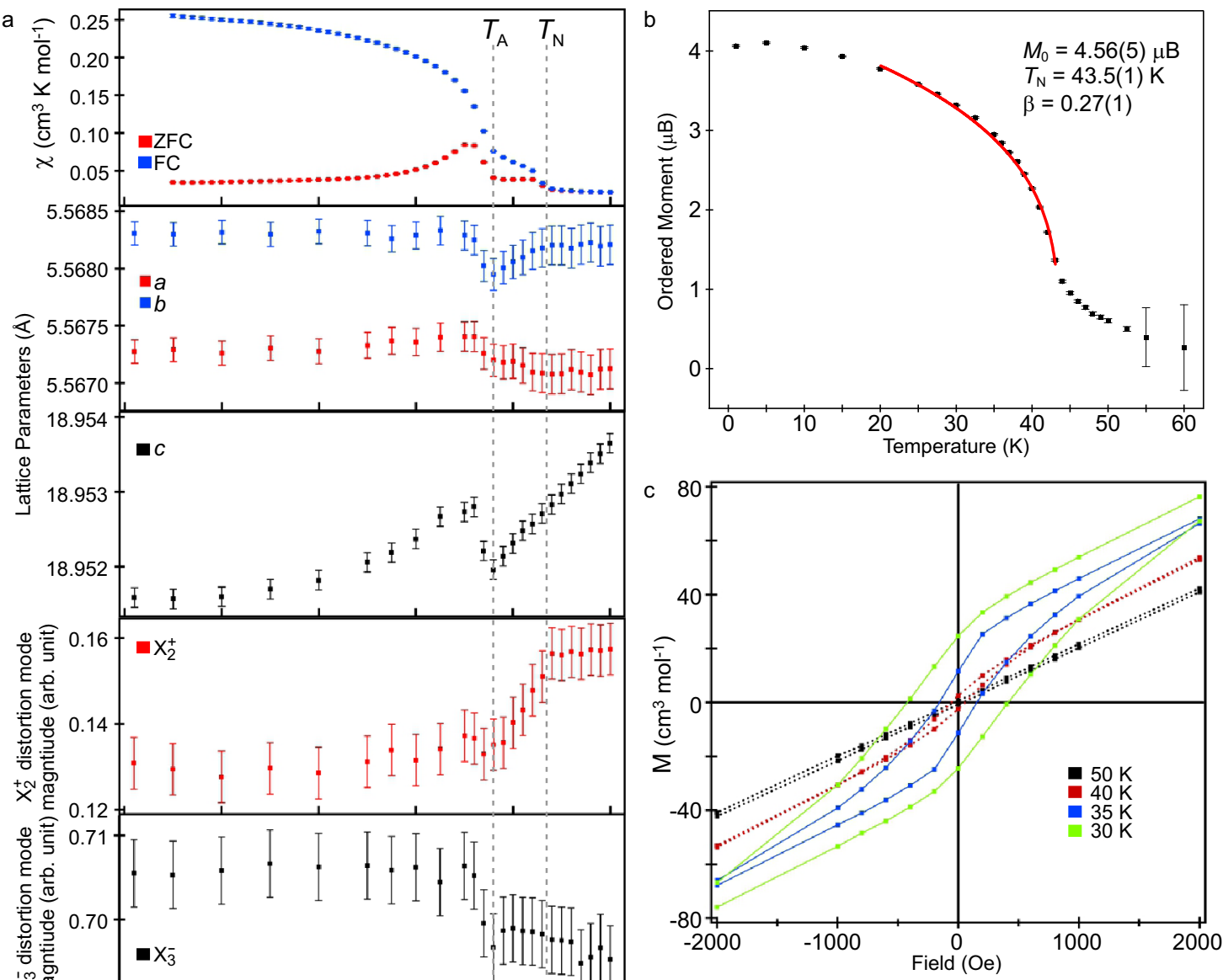

Fig. 4 Evidence for magnetoelectric coupling in $\mathbf{M n S r T a}_{\mathbf{2}} \mathbf{O}_{\mathbf{7}}$. a Zero-field-cooled and field-cooled magnetisation data, lattice parameters, $\mathrm{X}_{2}{ }^{+}, \mathrm{X}_{3}{ }^{-}$, and $\Gamma_{5}{ }^{-}$ distortion-mode magnitude (arbitrary units) 45,46 and $\mathrm{Mn}$ and $\mathrm{Sr} \Gamma_{5}{ }^{-}$mode displacement of $\mathrm{MnSrTa}_{2} \mathrm{O}_{7}$ in the temperature range $0<\mathrm{T} / \mathrm{K}<50$. b $M=M_{0}\left(1-\frac{T}{T_{N}}\right)^{\beta}$ power law fit to ordered Mn moment (obtained by NPD data) as a function of temperature. $\mathbf{c}$ Expanded region around $\mathrm{H}=0$ of magnetisation-field data collected from $\mathrm{MnSrTa}_{2} \mathrm{O}_{7}$ in an applied field in the range $-50000<\mathrm{H} / \mathrm{Oe}<50000$.

of the exponential term obtained from the power-law fit $(\beta=0.27(1))$ is in excellent agreement with other systems that show strong $2 \mathrm{D}$ magnetic interactions, but weak $3 \mathrm{D}$ coupling ${ }^{40}$.

As noted above, the ZFC and FC magnetisation data diverge at $T_{\mathrm{N}}=43 \mathrm{~K}$ (Fig. 4a). However, these data show a further feature at $38 \mathrm{~K}$, marked $T_{\mathrm{A}}$, where the gradient of the ZFC and FC data increases sharply. Field-dependent magnetisation data (Fig. 4c) collected at $40 \mathrm{~K}$, (between $T_{\mathrm{N}}$ and $T_{\mathrm{A}}$ ) are nonlinear and exhibit weak hysteresis, consistent with a canted antiferromagnetic state below $T_{\mathrm{N}}$. Equivalent data collected at $35 \mathrm{~K}$ and $30 \mathrm{~K}$ (below $T_{\mathrm{A}}$ ) show a significant increase in both remnant magnetisation and coercive field consistent with a sharp increase in the ferromagnetic component (increased canting) of the magnetic state.

Signatures of the event at $T_{\mathrm{A}}$ are also observed in the crystallographic data, with both the $b$ and $c$ lattice parameters exhibiting sharp minima at this temperature (Fig. 4a). A more detailed understanding of the structural changes occurring around $T_{\mathrm{N}}$ and $T_{\mathrm{A}}$ can be gained by analysing the changes to the magnitudes of the tilting distortion modes, $\mathrm{X}_{3}^{-}$and $\mathrm{X}_{2}{ }^{+}$, and the polar distortion mode, $\Gamma_{5}^{-}$, which relates the $a^{-} a^{-} c^{+} / a^{-} a^{-} c^{+}$distorted structure of $\mathrm{MnSrTa}_{2} \mathrm{O}_{7}$ to the corresponding undistorted aristotype structure. 
The $\mathrm{X}_{2}{ }^{+}$mode, which corresponds to the $a^{0} a^{0} c^{+} / a^{0} a^{0} c^{+}$tilting distortion of the $\mathrm{TaO}_{6}$ octahedra, is temperature independent from $50 \mathrm{~K}$ to $T_{\mathrm{N}}$ and then exhibits an almost linear decline of around $15 \%$ from $T_{\mathrm{N}}$ to $T_{\mathrm{A}}$, before becoming essentially temperature independent below $T_{\mathrm{A}}$, as shown in Fig. 4a. This suggests that while the $\mathrm{X}_{2}{ }^{+}$mode responds to the onset of magnetic order at $T_{\mathrm{N}}$, changes to the magnitude of this mode are not responsible for the minima in the lattice parameters at $T_{\mathrm{A}}$.

In contrast, the $\mathrm{X}_{3}{ }^{-}$mode and the $\Gamma_{5}{ }^{-}$polar distortion mode are essentially temperature independent between $50 \mathrm{~K}$ and $T_{\mathrm{A}}$, and thus do not appear to respond to the onset of magnetic order at $T_{\mathrm{N}}$. However, on cooling below $T_{\mathrm{A}}$, both modes show a step change: an increase of $\sim 1 \%$ in $\mathrm{X}_{3}{ }^{-}$and a sharp drop of around $5 \%$ in the magnitude of the $\Gamma_{5}{ }^{-}$mode, the latter change indicating a drop in the electrical polarisation of $\mathrm{MnSrTa}_{2} \mathrm{O}_{7}$ at this temperature (Fig. 4a). The drop in the magnitude of the $\Gamma_{5}{ }^{-}$mode can also be seen in sharp changes to the polar displacements of the $\mathrm{Mn}$ and Sr cations, also shown in Fig. 4a. The polar displacement of the $\mathrm{Sr}$ cations increases, while that of the Mn cations decreases, because the displacements of the $\mathrm{Mn}$ cations in the $\Gamma_{5}{ }^{-}$distortion mode oppose the establishment of a net polarisation as $\mathrm{MnSrTa}_{2} \mathrm{O}_{7}$ can be thought of as a ferrielectric rather than a ferroelectric.

Taking all these observations together, we observe that at 300 $\mathrm{K}, \mathrm{MnSrTa}_{2} \mathrm{O}_{7}$ adopts an incommensurate polar structure (although we note the incommensurate modulation of the structure is not the origin of the inversion symmetry breaking) in a paramagnetic state. On cooling below $T \sim 100 \mathrm{~K}$, 2-dimensional magnetic correlations build up, before the system undergoes a transition to 3-dimensional magnetic order at $T_{\mathrm{N}}=43 \mathrm{~K}$. On cooling to $T_{\mathrm{A}}=38 \mathrm{~K}$, there is a further transition in which the magnitude of the ferromagnetic moment of the phase increases, the $\mathrm{X}_{3}{ }^{-}$distortion increases, and the $\Gamma_{5}{ }^{-}$polar mode decreases.

A symmetry analysis of $\mathrm{MnSrTa}_{2} \mathrm{O}_{7}$ (see Supplementary Information) shows that the $\mathrm{m}_{5}{ }^{+}$weak ferromagnetic moment (which arises from the trilinear invariant of the primary antiferromagnetic $\mathrm{mX}_{3}{ }^{+}$mode and the $\mathrm{X}_{2}{ }^{+}$octahedral tilting) is directly coupled to the $\Gamma_{5}{ }^{-}$(HIP) polarisation through a quadrilinear invariant involving the $\mathrm{m \Gamma}_{5}^{+}, \Gamma_{5}^{-}, \mathrm{mX}_{3}{ }^{+}$and the $\mathrm{X}_{3}{ }^{-}$ octahedral tilting. Data in Figs. 4a, b show that the magnitudes of the $\Gamma_{5}{ }^{-}, \mathrm{mX}_{3}{ }^{+}$, and $\mathrm{X}_{3}{ }^{-}$distortions undergoe a step change at $T_{\mathrm{A}}$, coincident with a sharp increase in the weak ferromagnetic moment $\left(\mathrm{m \Gamma}_{5}{ }^{+}\right)$of $\mathrm{MnSrTa}_{2} \mathrm{O}_{7}$, consistent with magnetoelectric coupling.

This quadrilinear coupling also gives an indication that it is possible to reverse both improper ferroic orders, leaving the energy of the system invariant. The actual switching mechanism is more complex and can include different paths, depending on the distortions involved. Indeed, the reversal of the improper ferroelectric polarisation, achievable by the application of an external electric field, requires the reversal of either the $\mathrm{X}_{3}{ }^{-}$or the $\mathrm{X}_{2}{ }^{+}$octahedral tilting due to the HIP mechanism. If the $\mathrm{X}_{2}^{+}$ octahedral tilting changes sign, this will also require the reversal of the antiferromagnetic $\mathrm{mX}_{3}{ }^{+}$mode or reversal of the weak ferromagnetic moment $\left(\mathrm{m}_{5}{ }^{+}\right)$, due to the trilinear invariant coupling of these distortions to the $\mathrm{X}_{2}{ }^{+}$tilting. This gives a clear path for the electrical control of the weak ferromagnetic moment via the $\mathrm{X}_{2}{ }^{+}$tilting. The reverse mechanism, the magnetic control of the electrical polarisation, is less likely since the application of a magnetic field to switch the weak ferromagnetic moment will presumably result in the change of sign of the $\mathrm{mX}_{3}{ }^{+}$antiferromagnetic mode and not in a change of the $\mathrm{X}_{2}{ }^{+}$tilting.

The symmetry analysis and couplings detailed above are analogous to those described by Benedek and Fennie for
$\mathrm{Ca}_{3} \mathrm{Mn}_{2} \mathrm{O}_{7}{ }^{16}$. However, the appearance of a weak ferromagnetic moment at $T_{\mathrm{N}}$ prior to the onset/enhancement of magnetoelectric coupling at $T_{\mathrm{A}}$ does not appear to have an analogue in the observed behaviour of $\mathrm{Ca}_{3} \mathrm{Mn}_{2} \mathrm{O}_{7}$. Accurate theoretical investigations and switching experiments will be needed to identify the details of the coupling mechanism.

It should be noted that $\mathrm{Mn}_{3} \mathrm{O}_{4}$ exhibits a magnetic ordering transition $\left(T_{\mathrm{N}} \sim 41-43 \mathrm{~K}\right)^{41,42}$ at a temperature similar to the magnetic events observed for $\mathrm{MnSrTa}_{2} \mathrm{O}_{7}$. However, as shown above, the only features observed in the magnetisation data collected from $\mathrm{MnSrTa}_{2} \mathrm{O}_{7}$ are observed at $T_{\mathrm{N}}$ and $T_{\mathrm{A}}$, which can be correlated to features in the NPD data collected from $\mathrm{MnSrTa}_{2} \mathrm{O}_{7}$ (Fig. 4) and attributed to symmetry-required changes of the weak ferromagnetic moment $\left(\mathrm{mr}_{5}{ }^{+}\right)$of $\mathrm{MnSrTa}_{2} \mathrm{O}_{7}$. Thus, we can conclude that even if small quantities of $\mathrm{Mn}_{3} \mathrm{O}_{4}$ are present in the sample of $\mathrm{MnSrTa}_{2} \mathrm{O}_{7}$, any contribution they may make to the magnetisation data does not affect the veracity of the analysis presented above. We emphasise that there is no sign of any binary manganese oxide phases in any of the diffraction data collected from any of the samples prepared.

In conclusion, a directed cation-exchange reaction, which replaces the diamagnetic $\mathrm{Li}^{+} \mathrm{A}$-cations of the HIP ferroelectric phase $\mathrm{Li}_{2} \mathrm{SrTa}_{2} \mathrm{O}_{7}$ with paramagnetic $\mathrm{Mn}^{2+}$ cations allows the preparation of a coupled magnetoelectric material, $\mathrm{MnSrTa}_{2} \mathrm{O}_{7}$. Given that this type of cation-exchange chemistry should be broadly applicable, this suggests that a wide variety of $\mathrm{M}^{2+}$ transition-metal cations can be substituted into polar $\mathrm{Li}_{2} \mathrm{AB}_{2} \mathrm{O}_{7}$ host phases, allowing the preparation of a large number of additional, potentially magnetoelectric, materials.

\section{Methods}

Synthesis. Polycrystalline samples of $\mathrm{Li}_{2} \mathrm{SrTa}_{2} \mathrm{O}_{7}$ were prepared by combining suitable stoichiometric ratios of $\mathrm{SrCO}_{3}(99.994 \%)$ and $\mathrm{Ta}_{2} \mathrm{O}_{5}(99.993 \%$, dried at $900{ }^{\circ} \mathrm{C}$ ) and a $10 \%$ stoichiometric excess of $\mathrm{Li}_{2} \mathrm{CO}_{3}(99.998 \%)$ to compensate for metal loss due to volatility at high temperature. This mixture was then heated at $600^{\circ} \mathrm{C}$ in air for $12 \mathrm{~h}$, reground, and pressed into pellets. The pellets were then heated to $1250{ }^{\circ} \mathrm{C}$ at a heating rate of $5{ }^{\circ} \mathrm{C} / \mathrm{min}$ and kept at $1250^{\circ} \mathrm{C}$ for $12 \mathrm{~h}$, followed by cooling at $5^{\circ} \mathrm{C} / \mathrm{min}$ to room temperature. Samples were then reground, pressed into pellets, and heated at $1250{ }^{\circ} \mathrm{C}$ for $3 \mathrm{~h}$, before being quenched to room temperature. Polycrystalline samples of $\mathrm{MnSrTa}_{2} \mathrm{O}_{7}$ were prepared by reacting $\mathrm{Li}_{2} \mathrm{SrTa}_{2} \mathrm{O}_{7}$ with 5-mole equivalents of anhydrous $\mathrm{MnCl}_{2}$ (98\%). The mixture was ground together in an agate pestle and mortar in an argon-filled glovebox, loaded in a Pyrex tube, and heated for four days at $375^{\circ} \mathrm{C}$ under flowing argon. The mixture was then washed with distilled water to remove the remaining chlorides, and dried for $12 \mathrm{~h}$ at $140{ }^{\circ} \mathrm{C}$ in air.

Characterisation. Reaction progress and the final sample purity were assessed using X-ray powder diffraction data collected using a PANalytical X'pert diffractometer incorporating an X'celerator position-sensitive detector (monochromatic $\mathrm{Cu} \mathrm{K} \alpha_{1}$ radiation). High-resolution synchrotron X-ray powder diffraction (SXRD) data were collected using instrument I11 at Diamond Light Source Ltd. using Si-calibrated X-rays with an approximate wavelength of $0.825 \AA$, from samples sealed in $0.3 \mathrm{~mm}$-diameter borosilicate glass capillaries. Low-temperature $\mathrm{X}$-ray powder diffraction data were collected using Rigaku Smartlab diffractometer fitted with a $\mathrm{Ge}$ crystal monochromator $\left(\mathrm{Cu}, \mathrm{K}_{\alpha 1}\right)$ and an Oxford Cryosystems Phenix cryostat. Neutron powder diffraction (NPD) data were collected on the HRPD and WISH diffractometers at the ISIS neutron source, with the samples contained in indium-sealed vanadium cans. Rietveld profile refinements were performed using JANA2006 $6^{43}$ and the GSAS suite of programs ${ }^{44}$. Symmetry analysis and distortion-model quantification was performed using the ISODISTORT software 45,46 .

Magnetisation data were collected using a Quantum Design MPMS-XL SQUID magnetometer. Zero-field-cooled (ZFC) and field-cooled (FC) data were collected in an applied field of 100 Oe. Powder second harmonic generation measurements were performed on a Kurtz-NLO system using a Nd:YAG laser with a wavelength of $1064 \mathrm{~nm}$. The SHG signal was recorded and compared with a standard sample of $\alpha-\mathrm{SiO}_{2}$. A detailed description of the experimental setup and process has been reported elsewhere ${ }^{36}$. 


\section{Data availability}

NPD data are available at https://doi.org/10.5286/ISIS.E.RB1910148. Other data are available on request to the authors.

Received: 10 May 2021; Accepted: 22 July 2021;

Published online: 16 August 2021

\section{References}

1. Eerenstein, W., Mathur, N. D. \& Scott, J. F. Multiferroic and magnetoelectric materials. Nature 442, 759 (2006).

2. Cheong, S. W. \& Mostovoy, M. Multiferroics: a magnetic twist for ferroelectricity. Nat. Mater. 6, 13-20 (2007).

3. Bibes, M. \& Barthelemy, A. Multiferroics: towards a magnetoelectric memory. Nat. Mater. 7, 425-426 (2008).

4. Scott, J. F. Data storage - Multiferroic memories. Nat. Mater. 6, 256-257 (2007).

5. Hill, N. A. Why are there so few magnetic ferroelectrics? J. Phys. Chem. B $\mathbf{1 0 4}$ 6694-6709 (2000).

6. Nye, F. J. Physical Properties of Crystals (Oxford University Press, 1957).

7. Halasyamani, P. \& Poeppelmeier, K. R. Noncentrosymetric oxides. Chem. Mater. 10, 2753-2769 (1998)

8. Pearson, R. G. The 2nd-order Jahn-Teller effect. THEOCHEM J. Mol. Struct. 12, 25-34 (1983).

9. Cohen, R. E. Origin of ferroelectricity in perovskite oxides. Nature 358, 136-138 (1992).

10. Kang, S. K., Tang, H. \& Albright, T. A. Structures for $\mathrm{d}^{0} \mathrm{Ml}_{6}$ and $\mathrm{Ml}_{5}$ Complexes. J. Am. Chem. Soc. 115, 1971-1981 (1993).

11. Kunz, M. \& Brown, I. D. Out-of-center distortions around octahedrally coordinated d $\mathrm{d}^{0}$ transition-metals. J. Solid State Chem. 115, 395-406 (1995).

12. Lefebvre, I. et al. Electronic-properties of antimony chalcogenides. Phys. Rev. Lett. 59, 2471-2474 (1987).

13. Lefebvre, I., Szymanski, M. A., Olivier-Fourcade, J. \& Jumas, J. C. Electronic structure of Tin monochalcogenides from SnO to SnTe. Phys. Rev. B 58, 1896-1906 (1998).

14. Seshadri, R. \& Hill, N. A. Visualizing the role of Bi 6s "Lone Pairs" in the offcenter distortion in ferromagnetic $\mathrm{BiMnO}_{3}$. Chem. Mater. 13, 2892-2899 (2001).

15. Stoltzfus, M. W., Woodward, P. M., Seshadri, R., Klepeis, J. H. \& Bursten, B. Structure and bonding in $\mathrm{SnWO}_{4}, \mathrm{PbWO}_{4}$, and $\mathrm{BiVO}_{4}$ : lone pairs vs inert pairs. Inorg. Chem. 46, 3839-3850 (2007).

16. Benedek, N. A. \& Fennie, C. J. Hybrid improper ferroelectricity: a mechanism for controllable polarization-magnetization coupling. Phys. Rev. Lett. 106, 107204 (2011)

17. Mulder, A. T., Benedek, N. A., Rondinelli, J. M. \& Fennie, C. J. Turning $\mathrm{ABO}_{3}$ antiferroelectrics into ferroelectrics: design rules for practical rotation-driven ferroelectricity in double perovskites and $\mathrm{A}_{3} \mathrm{~B}_{2} \mathrm{O}_{7}$ Ruddlesden-Popper compounds. Adv. Funct. Mater. 23, 4810-4820 (2013).

18. Benedek, N. A., Mulder, A. T. \& Fennie, C. J. Polar octahedral rotations: a path to new multifunctional materials. J. Solid State Chem. 195, 11-20 (2012).

19. Bousquet, E. et al. Improper ferroelectricity in perovskite oxide artificial superlattices. Nature 452, 732-736 (2008).

20. Benedek, N. A. Origin of ferroelectricity in a family of polar oxides: the DionJacobson phases. Inorg. Chem. 53, 3769-3777 (2014).

21. Oh, Y. S., Luo, X., Huang, F. T., Wang, Y. Z. \& Cheong, S. W. Experimental demonstration of hybrid improper ferroelectricity and the presence of abundant charged walls in (Ca, Sr) $)_{3} \mathrm{Ti}_{2} \mathrm{O}_{7}$ crystals. Nat. Mater. 14, 407-413 (2015).

22. Yoshida, S. et al. Hybrid improper ferroelectricity in $(\mathrm{Sr}, \mathrm{Ca})_{3} \mathrm{Sn}_{2} \mathrm{O}_{7}$ and beyond: universal relationship between ferroelectric transition temperature and tolerance factor in $n=2$ Ruddlesden-Popper phases. J. Am. Chem. Soc. 140, 15690-15700 (2018).

23. Yoshida, S. et al. Ferroelectric $\mathrm{Sr}_{3} \mathrm{Zr}_{2} \mathrm{O}_{7}$ : competition between hybrid improper ferroelectric and antiferroelectric mechanisms. Adv. Funct. Mater. 28, 1801856 (2018).

24. Liu, M. F. et al. Direct observation of ferroelectricity in $\mathrm{Ca}_{3} \mathrm{Mn}_{2} \mathrm{O}_{7}$ and its prominent light absorption. Appl. Phys. Lett. 113, 022902 (2018).

25. Zhu, T. et al. Theory and neutrons combine to reveal a family of layered perovskites without inversion symmetry. Chem. Mater. 29, 9489-9497 (2017).

26. Zhu, T., Gibbs, A. S., Benedek, N. A. \& Hayward, M. A. Complex structural phase transitions of the hybrid improper polar Dion-Jacobson oxides $\mathrm{RbNdM}_{2} \mathrm{O}_{7}$ and $\mathrm{CsNdM}_{2} \mathrm{O}_{7}(\mathrm{M}=\mathrm{Nb}, \mathrm{Ta})$. Chem. Mater. 32, 4340-4346 (2020).

27. Zhu, T. et al. Cation exchange as a mechanism to engineer polarity in layered perovskites. Chem. Mater. 30, 8915-8924 (2018).

28. Asaki, S. et al. Ferroelectricity of Dion-Jacobsen layered perovskites $\mathrm{CsNdNb}_{2} \mathrm{O}_{7}$ and $\mathrm{RbNdNb}_{2} \mathrm{O}_{7}$. Jpn. J. Appl. Phys. 59, 6 (2020).
29. Lobanov, M. V. et al. Crystal and magnetic structure of the $\mathrm{Ca}_{3} \mathrm{Mn}_{2} \mathrm{O}_{7}$ Ruddlesden-Popper phase: neutron and synchrotron X-ray diffraction study. J. Phys. Condens. Matter 16, 5339-5348 (2004).

30. Zhu, W. K., Pi, L., Huang, Y. J., Tan, S. \& Zhang, Y. H. Electrically induced decrease of magnetization in $\mathrm{Ca}_{3} \mathrm{Mn}_{2} \mathrm{O}_{7}$. Appl. Phys. Lett. 101, 192407 (2012).

31. Pitcher, M. J. et al. Tilt engineering of spontaneous polarization and magnetization above $300 \mathrm{~K}$ in a bulk layered perovskite. Science 347, 420-424 (2015).

32. Mallick, S. et al. Polar structures of $\mathrm{KNdNb}_{2} \mathrm{O}_{7}$ and $\mathrm{KNdTa}_{2} \mathrm{O}_{7}$. Chem. Mater 32, 7965-7972 (2020)

33. Ranmohotti, K. G. S., Josepha, E., Choi, J., Zhang, J. X. \& Wiley, J. B. Topochemical manipulation of perovskites: low-temperature reaction strategies for directing structure and properties. Adv. Mater. 23, 442-460 (2011).

34. Schaak, R. E. \& Mallouk, T. E. Perovskites by design: a toolbox of solid-state reactions. Chem. Mater. 14, 1455-1471 (2002).

35. Hayward, M. A. In Comprehensive Inorganic Chemistry II Vol. 2 (eds Reedijk, J. \& Poeppelmeier, K. R.) 417-453 (Elsevier, 2013).

36. Ok, K. M., Chi, E. O. \& Halasyamani, P. S. Bulk characterization methods for non-centrosymmetric materials: second-harmonic generation, piezoelectricity, pyroelectricity, and ferroelectricity. Chem. Soc. Rev. 35, 710-717 (2006).

37. Pagnier, T. et al. Phase transition in the Ruddlesden-Popper layered perovskite $\mathrm{Li}_{2} \mathrm{SrTa}_{2} \mathrm{O}_{7}$. J. Solid State Chem. 182, 317-326 (2009)

38. Petricek, V., Vanderlee, A. \& Evain, M. On the use of Crenel functions for occupationally modulated structures. Acta Cryst. Sect. A 51, 529-535 (1995).

39. van Smaalen, S. Incommensurate Crystallography (Oxford University Press, 2007).

40. Bramwell, S. T. \& Holdsworth, P. C. W. Magnetization and universal subcritical behavior in 2-dimensional XY magnets. J. Phys. Condens. Matter 5, L53-L59 (1993)

41. Guillou, F., Thota, S., Prellier, W., Kumar, J. \& Hardy, V. Magnetic transitions in $\mathrm{Mn}_{3} \mathrm{O}_{4}$ and an anomaly at $38 \mathrm{~K}$ in magnetization and specific heat. Phys. Rev. B 83, 094423 (2011).

42. Jensen, G. B. \& Nielsen, O. V. Magnetic strucutre of $\mathrm{Mn}_{3} \mathrm{O}_{4}$ (Hausmannite) between $4.7 \mathrm{~K}$ and Neel point, 41 K. J. Phys. C. Solid State Phys. 7, 409-424 (1974).

43. Petricek, V., Dusek, M. \& Palatinus, L. Crystallographic computing system JANA2006: general features. Z. Fur Kristallographie-Crystalline Mater. 229, 345-352 (2014).

44. Los Alamos National Laboratory. General Structure Analysis System. Los Alamos National Laboratory Report LAUR 86-748 (2000).

45. Campbell, B. J., Stokes, H. T., Tanner, D. E. \& Hatch, D. M. ISODISPLACE: a web-based tool for exploring structural distortions. J. Appl. Crystallogr. 39, 607-614 (2006).

46. ISOTROPY Software Suite (iso.byu.edu, 2007)

\section{Acknowledgements}

Experiments at the Diamond Light Source were performed as part of the Block Allocation Group award "Oxford Solid State Chemistry BAG to probe compositionstructure-property relationships in solids" (EE13284). Experiments at the ISIS pulsed neutron facility were supported by a beam time allocation from the Science and Technology Facilities Council (RB 1910148, DOI:10.5286/ISIS.E.RB1910148). PSH and WZ thank the Welch Foundation (Grant E-1457) for support. We thank Dr. Gavin Stenning for help with the collection of the low-temperature X-ray diffraction data in the Materials Characterisation Laboratory at the ISIS Neutron and Muon Source.

\section{Author contributions}

$\mathrm{TZ}$ prepared the samples and performed the bulk of the structural characterisation; FO performed the symmetry analysis, PM and ASG assisted with the NPD data collection and analysis; WZ and PSH performed the SHG measurements; MAH conceived and supervised the project and wrote the paper.

\section{Competing interests}

The authors declare no competing interests.

\section{Additional information}

Supplementary information The online version contains supplementary material available at https://doi.org/10.1038/s41467-021-25098-1.

Correspondence and requests for materials should be addressed to M.A.H.

Peer review information Nature Communications thanks the anonymous reviewer(s) for their contribution to the peer review of this work.

Reprints and permission information is available at http://www.nature.com/reprints

Publisher's note Springer Nature remains neutral with regard to jurisdictional claims in published maps and institutional affiliations. 
(c) (i) Open Access This article is licensed under a Creative Commons Attribution 4.0 International License, which permits use, sharing, adaptation, distribution and reproduction in any medium or format, as long as you give appropriate credit to the original author(s) and the source, provide a link to the Creative Commons license, and indicate if changes were made. The images or other third party material in this article are included in the article's Creative Commons license, unless indicated otherwise in a credit line to the material. If material is not included in the article's Creative Commons license and your intended use is not permitted by statutory regulation or exceeds the permitted use, you will need to obtain permission directly from the copyright holder. To view a copy of this license, visit http://creativecommons.org/ licenses/by/4.0/.

(C) The Author(s) 2021 